\title{
Desarrollo de Competencias Avanzadas en Computación en la Formación de los Arquitectos Latinoamericanos del Siglo XXI
}

Advanced Computing Competence Development in 2lst-century Latin American Architects' Education

\author{
> Luis Felipe González Böhme \\ Universidad Técnica Federico Santa María \\ Departamento de Arquitectura \\ luisfelipe.gonzalez@usm.cl
}

\author{
> Cristián Calvo Barentin \\ Universidad Técnica Federico Santa María \\ Departamento de Arquitectura \\ cristian.calvo@usm.cl
}

\begin{abstract}
Automation and robotics are increasingly penetrating all types of industries in developed countries including architecture, i.e., products and services related to architectural practice. Therefore, more than ever, architects, designers and artists are interested in developing computational thinking skills to be able to integrate more functionality into their creations and take direct control of their fabrication. But what can a small school of architecture in Latin America do to prevent the deskilling of its graduates and, instead, create new labor opportunities for them abroad. Third-year students integrate physical computing with visual programming in an active learning environment to develop free proposals.
\end{abstract}

Keywords: Architectural education; Physical computing; Visual programming; Computational thinking; Active learning

\section{Introducción}

Basten tres hechos, aparentemente aislados, para describir parcialmente el escenario al que nos enfrentamos. Primero, los robots industriales alcanzaron en 2013 el máximo histórico de ventas en el mundo con 168.000 unidades (Baroncelli, 2014). Segundo, el Internet de las Cosas (Internet of Things, en inglés) evoluciona rápidamente hacia la creación de las así llamadas Ciudades Inteligentes (Smart o Ubiquitous Cities, en inglés) como Songdo o Masdar y la transformación de otras como Viena, Amsterdam, Copenhagen o Santander. En ellas, una variedad de objetos (automóviles, edificios, vestuario, etc.) podrá interactuar y cooperar entre sí sin necesidad de intervención humana. Tercero, la Tercera Revolución Industrial (Rifkin, 2011) -avalada por el Parlamento Europeo (EP, 2011)demanda, i.a., que todos los edificios del planeta se transformen en micro-plantas de energía limpia, capaces de recoger y reaprovechar las energías renovables localmente. Aquí el factor común es el rápido desarrollo científico y tecnológico de la computación y su ubicuidad. En este escenario, el diseño y la construcción -incluso el habitar- del espacio arquitectónico se vuelven cada vez más complejos, hasta el punto de requerir métodos y modelos computacionales para razonar con el creciente volumen de información atinente y satisfacer la variedad de restricciones impuestas por nuevos estándares globales de eficiencia y de calidad. La exigencia, por lo tanto, para todo profesional del siglo XXI es dominar técnicas y aplicaciones de la computación, que le permitan resolver problemas y desarrollar proyectos en su campo de acción. Así, el desarrollo de competencias avanzadas en computación se ha vuelto un objetivo global en la formación de profesionales productivos y de calidad en esta nueva era. En los países desarrollados, la automatización y la robótica penetran progresivamente todo tipo de industrias, incluyendo la arquitectura, ya sea en la producción de obras o la prestación de servicios relacionados. Tanto Brell-Çokcan y Braumann (2013) como McGee y Ponce de León (2014) dan cuenta del creciente interés de arquitectos, diseñadores y artistas por los robots industriales -interés impulsado por el trabajo seminal de los arquitectos Gramazio y Kohler a principios de los 2000. En los países emergentes, en cambio, la introducción de dicha tecnología representa un reto en muchas áreas, no solo en arquitectura, también en la educación, producción, comercio, servicios, legislación, etc. Aunque aún es discutible si la automatización abre un camino hacia el desarrollo económico de un país, es indudable que oponerse hoy a su implantación cierra uno. Para los arquitectos y diseñadores la automatización tiene un "costo" que, según Woodbury (2010), implica un cambio radical en su modo de pensar y hacer. Nuestra visión -la que venimos anunciando desde hace un par de ańos ya (González \& Calvo, 2012; González, Calvo, \& Chiarella, 2013; González, Maino, Carrasco, \& Solís, 2012)-, es que también los arquitectos latinoamericanos del siglo XXI requerirán dominar las innovaciones científico-tecnológicas en su campo de acción profesional y aportar al mundo una perspectiva propia desde sus realidades e idiosincrasias. Las principales áreas de innovación en el campo de acción profesional de los arquitectos actualmente incluyen el diseño paramétrico basado en restricciones, la automatización y la robótica en la construcción, el diseńo de entornos inteligentes y la 


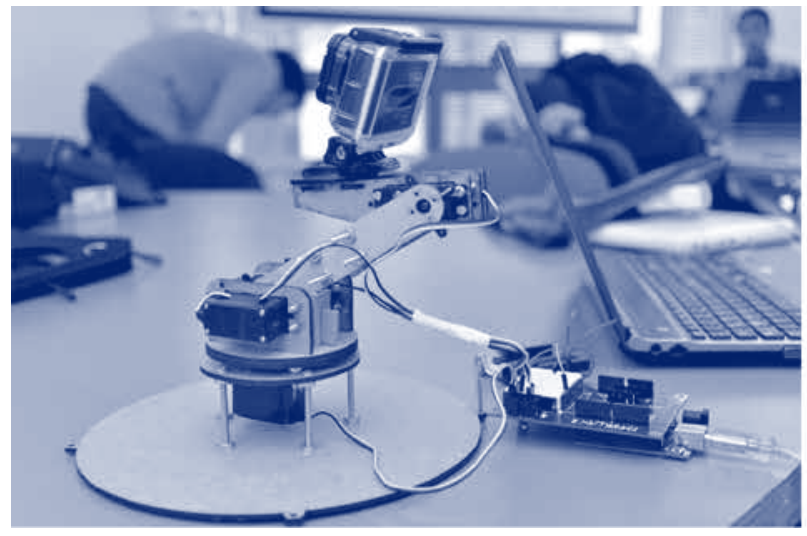

Figura 1: Los estudiantes integran bits y átomos con sus propias manos, en el Taller de Métodos Computacionales en Arquitectura 2013.

planificación de ciudades inteligentes (a veces llamado Urbanismo Digital), entre otras. En la formación de los futuros arquitectos entonces es necesario desarrollar una comprensión científico-tecnológica de la práctica arquitectónica, incentivarles a tomar el control de los nuevos procesos de producción de la forma construida, a incorporar en sus diseños diversidad, adaptabilidad y capacidad de respuesta a requerimientos variables y también a integrar la participación activa del usuario en los procesos de diseńo. La pregunta ante un escenario como el descrito aquí, es: ¿Qué estamos haciendo en las escuelas de arquitectura de América Latina, para evitar una potencial descualificación de nuestros egresados y, en cambio, crear para ellos nuevas oportunidades laborales en cualquier lugar del planeta?

En este artículo presentamos la asignatura llamada Taller de Métodos Computacionales en Arquitectura, dirigida a estudiantes de tercer año de la carrera de arquitectura de la Universidad Técnica Federico Santa María (UTFSM). Además comentamos los resultados de su primera versión, impartida durante el primer semestre de 2013, y de su segunda versión, impartida durante el primer semestre de 2014.

\section{El taller de métodos computacionales en arquitectura}

La actualización del plan de estudios de la carrera de arquitectura de la UTFSM, en 2012, creó una oportunidad para introducir nuevos contenidos y métodos de aprendizaje. Así se incorporaron dos nuevas asignaturas, obligatorias para cuarto y quinto semestres de la carrera, enfocadas al aprendizaje de métodos computacionales y de habilidades y actitudes que contribuyen al desarrollo de lo que Wing (2006) define como pensamiento computacional. En ambas asignaturas se aplican métodos de aprendizaje activo (Huber, 2008) y computación fisica (O'Sullivan $\&$ Igoe, 2004), en espacios destinados especialmente a ese uso. En la primera asignatura, llamada Métodos Computacionales en Arquitectura -descrita en (González, Calvo, \& Chiarella, 2013)-, se ensamblan y programan modelos a escala de sistemas de control automático y robótica móvil autónoma, con el objeto de aprender conceptos básicos de la programación de computadores tales como variables, tipos de datos, sentencias, uso de operadores aritméticos, operadores booleanos, comparadores, condicionales



y otros. Paralelamente, se pide a los estudiantes formular de manera estructurada un problema del "mundo real" local que les preocupe y un plan de solución que emplee automatización, basándose en los modelos a escala que ellos mismos ensamblan y programan. En la segunda asignatura, llamada Taller de Métodos Computacionales en Arquitectura, los estudiantes desarrollan en equipo pequeños proyectos de libre inspiración, que involucran diseño de interacción -desde el perspectiva de los diseñadores (Crampton-Smith, 2008)-, utilizando placas computadoras, actuadores, sensores y toda clase de materiales para maquetar. Aquí nuestros estudiantes de arquitectura experimentan, por primera vez, lo que es integrar bits y átomos (Negroponte, 1995) con sus propias manos (Figura 1). Creemos que esta asignatura contribuye al perfil de egreso de la carrera, estimulando el desarrollo de competencias específicas tales como:

- Dominar y aplicar conocimientos científico-tecnológicos avanzados para el diseño, evaluación y desarrollo del hábitat construido.

- Construir un diálogo activo interdisciplinario con las ciencias de la ingeniería.

- Y también, mediante el desarrollo de competencias transversales tales como:

- Pensamiento computacional

- Creatividad orientada a la resolución de problemas

- Visión tecnológica de la disciplina

- Manejo de problemas no estructurados

Se espera que luego de aprobar los estudiantes sean capaces de:

- Visualizar oportunidades para la arquitectura en la aplicación de métodos y modelos computacionales.

- Formular problemas de arquitectura de manera que puedan ser resueltos mediante la computación y otras herramientas.

- Representar datos relevantes de un proyecto de arquitectura mediante abstracciones, como modelos y simulaciones.

- Automatizar tareas relacionadas con la práctica de la arquitectura mediante pensamiento algorítmico.

El nombre de esta asignatura obedece al método de aprendizaje activo aplicado, que emula el del taller de arquitectura tradicional. Se trabaja principalmente en clase, hay correcciones de tablero y cuelgas para presentar avances de los proyectos, así como una presentación final, que incluye un video. Además se imparten algunas clases expositivas para tratar contenidos teóricos como los 

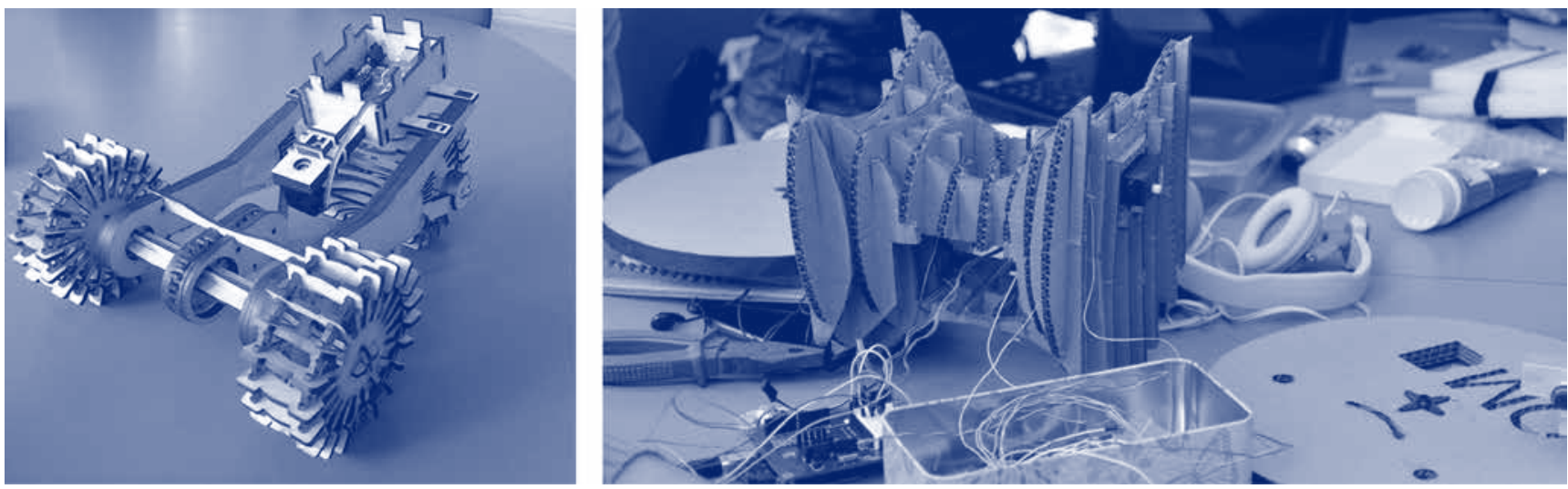

Figura 2: Robot sembrador y robot móvil con centro de masa desplazado, Taller de Métodos Computacionales en Arquitectura 2013.

principios básicos de la electrotecnia, diseño paramétrico y diseño basado en restricciones, que incluyen definiciones, ejemplos y ejercicios prácticos. Los estudiantes pueden utilizar también nuestro Laboratorio de Materialidad y Fabricación Digital (LaboMAT) para fabricar prototipos por control numérico, utilizando cortadora laser y fresadora.

La asignatura dispone un blog en donde los estudiantes comparten dudas, trucos y resultados. Para apoyar el desarrollo de los proyectos, contamos con un número modesto de placas computadoras ARDUINO ${ }^{\mathrm{TM}}$ UNO R3, placas BOE Parallax ${ }^{\mathrm{TM}}$ y algunos actuadores y sensores. Cada estudiante reconoce rápidamente la necesidad de equiparse con una "caja de herramientas" e insumos como cables, soldadura, etc.

\section{¿De qué sirve la computación en arquitectura?}

Al iniciar la primera asignatura, Métodos Computacionales en Arquitectura, se les pregunta a los estudiantes de cuarto semestre de la carrera, por la utilidad de la computación en arquitectura. La pregunta parece necia, igualmente si se hiciera en medicina, arqueología o leyes. Es, sin embargo, la pregunta de investigación fundamental en computación arquitectónica (architectural computing) y, como verán luego, para los arquitectos en formación, responderla no es trivial. El porcentaje de recurrencia de determinadas funciones en el total de respuestas recibidas fue el siguiente: visualizar/representar/3D (31\%), simular (27\%), dibujar planos (16\%), generar/producir/ crear (12\%), evaluar/analizar (7\%), logística (4\%) y calcular (3\%). A simple vista, se advierte que la representación gráfica sigue encabezando la lista de aplicaciones de la computación en arquitectura, como es tradicional. Le sigue la simulación de fenómenos físicos como el comportamiento térmico, que la sustentabilidad ambiental ha puesto de moda últimamente. Sorprende -no realmente- que la documentación planimétrica anteceda a la generación de alternativas de diseño y que aplicaciones como evaluación, logística y cálculo sean mencionadas apenas 5, 3 y 2 veces respectivamente en un total de 28 respuestas. Destaca el hecho que los estudiantes sólo identifiquen aplicabilidad de la computación en tareas relacionadas con el diseńo, quedando fuera cualquier otra etapa del ciclo de vida del entorno construido como su construcción, ocupación o desmantelamiento. Tampoco reconocen su aplicabilidad en otras tareas relacionadas con la práctica de la arquitectura $(\mathrm{CACH}, 2007)$ como programación arquitectónica, inspección técnica de obras, levantamientos, restauración, administración de obras, tasación de propiedades, etc.

\section{Proyectos de libre inspiración}

Al iniciar la segunda asignatura, el Taller de Métodos Computacionales en Arquitectura, los estudiantes ya tienen una noción de cómo es la arquitectura básica de algunos sistemas simples de control automático y robots móviles autónomos y cómo programarlos. Entonces se les encarga diseñar e implementar prototipos de objetos o entornos interactivos, empleando esta vez herramientas de computación física más poderosas y flexibles. En la primera versión de esta asignatura temimos que la etapa de ideación demoraría demasiado tiempo y por eso propusimos a los estudiantes desarrollar un brazo robótico. Solo dos equipos hicieron caso al de proyectos, todas interesantes aunque algunas demasiado complicadas de implementar con el nivel de conocimiento adquirido hasta ese momento. Las reglas del taller permiten, en todo caso, "construir sobre las ideas de otros" (Brown, 2009, p. 81; p. 239) y adaptarlas, siempre y cuando se reconozca la autoría. Los estudiantes se valieron de todos los medios a su alcance, probaron y quemaron componentes electrónicos, pidieron ayuda a compańeros de las carreras de ingeniería electrónica e informática, revisaron foros en Internet y sitios wiki, fabricaron piezas con la cortadora laser y a mano, etc. Así surgieron, i.a., un robot sembrador, un robot móvil de dos ruedas (Figura 2), un quitasol auto-orientable y un robot limpiavidrios para edificios en altura.

\section{Modificaciones a la segunda versión}

Aprendimos la lección y en la segunda versión del taller fomentamos desde un principio la libre inspiración de los estudiantes para idear toda clase de propuestas. Sólo que esta vez agregamos algunas herramientas que facilitan la integración de la programación de computadores y la modelación geométrica como el software de modelación 3D RHINOCEROS ${ }^{\mathrm{TM}}$, el entorno de programación visual GRASSHOPPER ${ }^{\mathrm{TM}}$ para parametrizar geometrías y la aplicación FIREFLY ${ }^{\mathrm{M}}$ de programación visual de placas computadoras ARDUINO $^{\mathrm{TM}}$ dentro del entorno GRASSHOPPER ${ }^{\mathrm{TM}}$. Esta encargo. Recibimos, en compensación, las más variadas propuestas 
modificación obedeció, en parte, a la persistente inquietud de los estudiantes por interactuar con software CAAD, como lo reflejaron las respuestas a la encuesta sobre la utilidad de la computación en arquitectura. El aprendizaje de estas herramientas lamentablemente consumió parte del tiempo destinado originalmente al desarrollo de los proyectos. A pesar de todo, un equipo de estudiantes nos sorprendió positivamente al intentar construir un sensor de flexión casero para un modelo elástico de un muro cuya deformación es graficada en tiempo real en el computador (Figura 3).

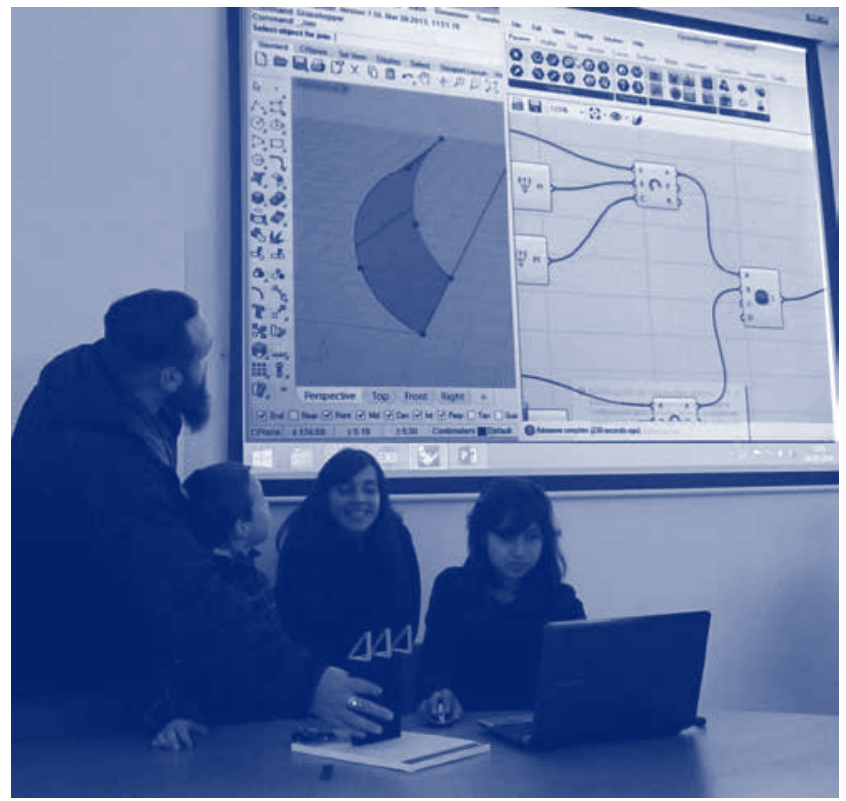

Figura 3: Proyecto Flexi-Muro para simulación sísmica mediante sensores de flexión integrados en los muros, 2014.

\section{¿Qué efecto ha provocado esta asignatura en su modo de pensar y actuar?}

En las encuestas que realizamos al finalizar cada asignatura, las distintas generaciones de estudiantes han calificado ambas versiones del Taller de Métodos Computacionales de manera similar, es decir, Entre Aceptable (3) y Bueno (4), en una escala que va de Malo (1) a Excelente (5). La excepción es que la valoración que los estudiantes hacen de la "Relevancia de la asignatura para la formación integral del profesional UTFSM" aumentó levemente $(-8 \%)$ en la segunda versión. Ante la pregunta que encabeza esta sección, los estudiantes contestaron lo siguiente:

"Este ramo te enseña a comprender, de manera simple, el funcionamiento de la mayoría de las tecnologias presentes hoy día en el mundo. También he planteado en mi mente muchas ideas de procesos de automatización en tareas cotidianas. Pienso que podría adquirir una placa ARDUINO ${ }^{\mathrm{Tm}}$ y empezar a probar por mi cuenta las tecnologías que se me presenten." (sic)

"La verdad es que no pensaba mucho en la utilidad que se le podia dar a la programación en la arquitectura, de hecho, no pensé ni siquiera en utilizarla en un futuro laboral. Este ramo me hizo revalorizar las herramientas y el hecho de implementarla en la arquitectura propia." (sic)

"Aumentó el interés por la tecnología aplicada a la arquitectura y a la vida diaria, y por buscar nuevas soluciones a problemas cotidianos." (sic)

"Ha despertado un poco las ganas en mí, de saber sobre robots en la arquitectura. Fue la primera aproximación en la carrera que he tenido hacia las áreas tecnológicas. Por lo tanto, creo que ha provocado un sentimiento de ganas de descubrir esta rama de la arquitectura." (sic)

\section{Conclusiones y discusión}

Todavía quedan colegas a quienes les preocupa que: "Nuestros estudiantes serán competentes en robots, programación, cálculo térmico y otras especificidades." (sic) porque eso “...podría conducir a una pérdida de liderazgo de nuestros egresados, ya que una vez más, nuestros titulados serán contratados por los que hacen la arquitectura en Chile (los arquitectos titulados por la Pontificia Universidad Católica y Universidad de Chile) para resolver la aplicación de un software, calcular la ganancia térmica en invierno o para desarrollar un robot que limpie los vidrios en una torre de oficinas." (sic). Entonces “¿quién velará por la tradición de la generalidad y el sentido común en la arquitectura?” (sic). Esta reflexión propone aislar al diseńo arquitectónico de cualquier otra práctica y conocimiento específico en la formación de los arquitectos para preservar su rol de generalistas. Nosotros creemos que ese camino conducirá irremediablemente a la descualificación de nuestros futuros egresados, al enfrentar un mundo altamente tecnologizado y comunicado. Por otra parte, observamos que la mayoría de nuestros estudiantes no necesariamente cuentan con todas las características atribuibles a los nativos digitales (Prensky, 2001), como lo prueban algunas de las respuestas a la pregunta ¿qué cambiaría de esta asignatura?:

"Que las entregas de tareas y trabajos no se realicen mediante internet (blog) ya que es muy enredado de utilizar." (sic)

"En la asignatura deberían ayudarnos a instalar los programas, ya que este año hubo problemas con esto." (sic)

"Deberian ayudar a los alumnos en la instalación de programas que se ocuparán durante el semestre para que las clases sean más expeditas y educativas desde un principio." (sic)

"Agregaría una clase en la cual todos instalen los programas (Rhino, Grasshopper, etc.), los cuales personalmente desconozco como instalar." (sic)

Finalmente, los estudiantes demostraron ser capaces de visualizar, diseñar y desarrollar proyectos de computación física simples, a partir de problemas y desafíos propios de la arquitectura. Por 
todo lo anterior, creemos que es necesario repensar la formación de los arquitectos en América Latina, integrando el aprendizaje del diseño arquitectónico con otras prácticas y conocimientos específicos como la computación.

\section{Reconocimientos}

Agradecemos la gentil colaboración del profesor Jorge Juliet del Departamento de Ingeniería Eléctrica de la UTFSM. El diseño e implementación de ambas asignaturas han sido financiadas con fondos del Estado Chileno, a través del programa para el Mejoramiento de la Calidad y Equidad de la Educación Superior (MECESUP FSM802) y fondos institucionales para la Renovación de Laboratorios de Computación y Materialidad 2014 (USM-491206).

\section{Referencias}

Baroncelli, A. (2014). All-time-high for industrial robots in 2013, Retrieved from http://www.worldrobotics.org/index.php?id=home\&news_id=272

Brell-Çokcan, S., \& Braumann, J. (Eds.). (2013). Robotic fabrication in architecture, art, and design. Vienna, Austria: Springer Verlag.

Brown, T. (2009). Change by design: How design thinking transforms organizations and inspires innovation. New York, USA: HarperBusiness.

CACH. (2007). Actos y servicios profesionales de los arquitectos. Retrieved from http://www.colegioarquitectos.com/DOCUMENTOS/legales/Arancel\%20Servs.pdf

Crampton-Smith, G. (2008). El oficio del diseño de interacción. ELISAVA TdD, 25(Investigación en diseño), pp. 13-19.

EP. (2011). European council: Post-carbon economy requires third industrial revolution. Brussels, Belgium: European Parliament.

González, L. F. \& Calvo, C. (2012, 7-10 Noviembre). ARQ232: La introducción de juegos de construcción \& robótica pedagógica en la formación de arquitectos con competencia en CyT. Libro de ponencias del 2 do. Encuentro Latinoamericano INTROArq: Introducción a la enseñanza de la arquitectura: Métodos, protagonistas y contexto, Valparaíso, Chile, pp. 334-344.

González, L. F., Calvo, C., \& Chiarella, M. (2013, 20-22 Noviembre). Métodos computacionales en arquitectura: la formación de arquitectos con competencia en CyT. Libro de ponencias del XVII Congreso SIGraDi, Valparaíso, Chile, pp. 524-528.

González, L. F., Maino, S., Carrasco, C., \& Solís, R. (2012, 10-12 Octubre). Juegos de construcción \& robótica pedagógica en la formación de arquitectos con competencia en CyT. Artículo presentado en el XXV Congreso de la Sociedad Chilena de Educación en Ingeniería (SOCHEDI), Antofagasta, Chile.

Huber, G. (2008). Aprendizaje activo y metodologías educativas. Revista de Educación, número extraordinario, pp. 59-81.

McGee, W., \& Ponce de Leon, M. (Eds.). (2014). Robotic Fabrication in Architecture, Art and Design 2014. New York, USA: Springer.

Negroponte, N. (1995). Being digital. New York, USA: Vintage Books.

O'Sullivan, D., \& Igoe, T. (2004). Physical computing: sensing and controlling the physical world with computers. Mason, USA: Course Technology Press.

Prensky, M. ( 2001). Digital Natives, Digital Immigrants. On the Horizon, 9(5), pp. 1-6.

Rifkin, J. (2011). La tercera revolución industrial: cómo el poder lateral está transformando la energía, la economía y el mundo. Barcelona, España: Ediciones Paidós.

Wing, J. (2006). Computational thinking. Communications of the ACM, 49(3), pp. 33-35.

Woodbury, R. (2010). Elements of parametric design. New York, USA: Routledge. 